\title{
Mallarmé et la poétique de l'hybridité
}

Tant de monstres parcourent les sous-bois de la poésie symboliste et presque toujours ce sont des hybrides; des corps d'ascendance mythologique et composites. Pourquoi si souvent des hybrides? Sans doute faut-il apprécier une fréquentation devenue familière sinon normalisée. Comme lécrit l’abbé Du Bos à propos de certains personnages allégoriques, ils «sont nés depuis plusieurs années; depuis longtemps ils ont fait fortune ; [...] ils ont acquis, pour ainsi dire, droit de bourgeoisie parmi le genre humain $»^{1}$. En revanche, ces figures qui se rencontrent encore volontiers dans les parcs et bosquets des jardins français du XVIII siècle comme autant d'agréments à la promenade, désignent plus franchement - un siècle plus tard - les secrets mêmes de l'activité poétique. La multiplication des occurrences hybrides dans la poésie symboliste témoigne en effet moins d'un quelconque intérêt dépoque en ce qui concerne les croisements d'espèces au sein de la nature quelle ne vise d'abord à éprouver la nature même de «l'art ». Les temps ne sont plus à la « querelle des monstres » et les hybrides de la modernité poétique - s'ils se disputent la scène mythologique de la poésie symboliste - s'y promènent en toute innocence. Pour autant, au-delà des facilités d'école, ce bestiaire mythologique n’est ni innocent ni suranné ; il incarne bien plutôt un véritable répertoire mythopoétique. Chez Mallarmé comme chez Nerval, Gautier, Baudelaire ou Rimbaud, les figures hybrides indiquent presque toujours une connivence avec la nature et le chant, la musique et l'art, l'activité créatrice. Ainsi, le faune soufflant dans sa flûte est-il un poète " qui semble animer les bois et faire danser les branches des arbres ». Poète, il lui appartient de recréer un monde " où

Dr Olivier Sécardin - titulaire d'un doctorat de littérature comparée obtenu en Sorbonne. Après avoir enseigné dans de nombreuses universités internationales, notamment à la Columbia University de New York, à l'Université du Kyushu (au Japon), à l'Université de Chicago ainsi qu'à Cornell University (ÉtatsUnis), il est actuellement professeur assistant de littérature française à l'Université d'Utrecht (Pays-Bas). Étudiant aussi bien les textes canoniques que les formes plus contemporaines, il est lui-même lauteur de textes littéraires et artistiques. Adresse pour correspondance : Department of Humanities, Utrecht University, Trans 10, 3512 JK, Utrecht, The Netherlands ; email : O.Secardin@uu.nl

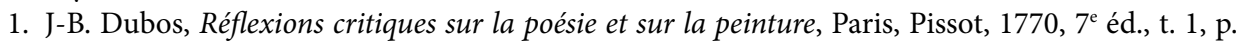
193. 
interviendrait plus quà demi comme sirènes confondues par la croupe avec le feuillage et les rinceaux d'une arabesque, la figure, que demeure l'idée ». Par analogie, l'art du poète, faune de la poésie, célèbre l' « Hymen » entre l'homme et le monde. En désignant une certaine opération de réflexivité vers laquelle le poème lui-même se fait signe - signe impossible mais nécessaire, à l'image du Livre mallarméen - l'hybridité est promue comme structure privilégiée de la modernité poétique.

\section{Portrait de l'artiste en hybride}

Si les hybrides sont légion au panthéon littéraire de Mallarmé, c'est qu'ils incarnent tous le génie créateur ${ }^{2}$. Chez Mallarmé, artistes et poètes sont effectivement évoqués sous les traits de figures hybrides. On se souvient du portrait de Mallarmé peint par Manet en 1876, ce " curieux tableautin » longuement commenté par Bataille (1955) et identifié par Valéry comme rencontre triomphale de la peinture et de la poésie. Cette amitié scelle une reconnaissance réciproque. Ainsi, dans ses Divagations (1897), Mallarmé décèle-t-il en Manet une « ingénuité de chèvre-pied au pardessus mastic, barbe et blond cheveu rare, grisonnant avec esprit $»^{3}$. Manet, créature sabotée ? Quelle signification attribuer à cette caractérisation elle-même hybride ? On se souvient du Docteur Bordeu de Diderot imaginant des chèvre-pieds comme domestiques et peut-être plus confidentiellement des vers plus anciens de Rémy Belleau : "A Dieu donc, garses forestières,/ A Dieu, pucelles fontainières,/ Chèvrepiés, satyres cornus,/ Faunes, sylvains et Dieux cornus » (1995: 37), surtout de nombreuses pastorales. Plus proche de Mallarmé, ceux de Victor Hugo dans La Légende des Siècles. Dans son article "Hugo et le chèvre-pied ", Jean-Bertrand Barrère ne manque pas de rappeler l'ambiguité du terme. Que désigne au juste le "chèvre-pied "? Pour Paul Berret cité par Jean-Bertrand Barrère, seuls les satyres et les ægipans ont droit à ce nom de chèvre-pied : "Les faunes sont représentés avec des jambes d'hommes " (1958 : 121). Littré indique également : " Dieux chèvre-pieds, les satyres ». Faune est le nom latin du protecteur des troupeaux, Satyre est le nom de la divinité grecque ; chèvre-pied, le composé français, serait une invention du XVI siècle. Les emplois sont en vérité très peu distingués. Hugo, par exemple, emploie indistinctement faune, satyre, ægipan, chèvre-pied et sylvain. Leurs corollaires féminins ne sont pas davantage spécifiés : nymphes, dryades et naïades séchangent leurs rôles. Quant au Manet de Mallarmé, il faut apprécier une sorte de virilité un peu rude et surtout un rapport de proximité avec la nature.

Dans L'Après-midi d'un faune, le faune se dit ingénu - une caractérisation tout aussi bien attachée à Verlaine, «l'enfant avec son ingénue audace marchant en l'existence

2. En particulier, Mattiussi (1998a : 141-159).

3. S. Mallarmé, "Édouard Manet ", Divagations, Euvres complètes, t. II, éd. Bertrand Marchal, Paris, Gallimard, coll. «Bibliothèque de la Pléiade», 2003, p. 146. [Désormais OC II ]. 
selon sa divinité » ${ }^{4}$; de son époque il " s'extrait avec ingénuité ${ }^{5}$ puisqu’il se trouve placé au "conflit de deux époques $»^{6}$. Citons de façon plus anecdotique ce poème de jeunesse qui fait de Pan une divinité tutélaire du poète :

Ô Pan! fais de ma voix la trompette fidèle

Qui jette à l’univers au milieu des éclairs

Un éclat de ta voix, un feu de ta lumière!

Fais de moi ton archange !... une aile dont les airs

Gardent la trace en feu comme de ton tonnerre ! ${ }^{7}$

Maupassant, pourtant monstre réaliste, est également évoqué sous les traits d'un " faune inné de la vision et du dire ». Cette récurrence du faune n’est pas particulière à Mallarmé. On la retrouve déjà chez Alfred de Musset (dans Rolla, en 1833), chez Victor Hugo, chez Leconte de Lisle (Poèmes antiques, en 1852) ou chez Rimbaud, par exemple. En 1937, Albert Schinz ${ }^{8}$ a également indiqué - en ce qui concerne Mallarmé - le probable intertexte de la "Diane au bois " (1864) de Théodore de Banville. Quoi qu'il en soit, le faune termine sa carrière en 1895 dans les "Offrandes à divers du faune » où il s'assimile à divers « créateurs » et surtout à Mallarmé lui-même :

Faune

avec ton chant s'il brusqua

Le cours de l'heure qui séloigne

Retiens près du Bois de Boulogne

La princesse Poniatowska

Sylvain d'haleine première

Si ta flûte a réussi

Ouïs toute la lumière

Qu'y soufflera Debussy

À motif que sa flûte file

Le Faune heureux le dédia

Sur Hollande au bibliophile

Et haut rimeur Hérédia. ${ }^{9}$

4. S. Mallarmé, « Verlaine », Médaillons et Portraits, Divagations, OC II, (2003 : 120).

5. S. Mallarmé, «Sur Verlaine », Proses diverses, OC II, (2003: 662).

6. Ibid.

7. S. Mallarmé, "Pan ", Poèmes de jeunessse, CEuvres complètes, t. I, éd. Bertrand Marchal, Paris, Gallimard, coll. «Bibliothèque de la Pléiade », 1998, p. 210. [Désormais OC I].

8. A. Schinz, "D’où sort l'Après-midi d’un faune de Mallarmé ? ", Modern Language Notes, vol. 52, nov. 1937.

9. S. Mallarmé, « Offrandes à divers du faune », Vers de circonstance, OC I, (1998:280). 
Il faut enfin considérer comme un calembour cette attribution pseudo-scientifique de Max Nordau : " Ajoutons encore, pour être complet, qu'on remarque chez Mallarmé des oreilles longues et pointues de satyre » $(1894: 232)$.

Parmi les figures dynamiques et intermédiaires du corpus mallarméen, celle du héros est particulièrement appuyée. Le héros est en effet un demi-dieu, à demi humain, à demi divin. Comme les hybrides, il se situe à l'intersection des règnes. Assurant la médiation entre les théoi et les mortels, le héros est médian. Or, chez Mallarmé la vertu de l'art est précisément d'assurer la médiation du «fait à l'Idéal ». Par l'opération de l'art, le poète se métamorphose en héros. Dans La Musique et les lettres, "Voter, même pour soi, ne contente pas, en tant qu'expansion d'hymne avec trompettes intimant l'allégresse de némettre aucun nom ; ni l'émeute, suffisamment, nenveloppe de la tourmente nécessaire à ruisseler, se confondre, et renaître, héros $»^{10}$. Laurent Mattiussi, en particulier, a montré que "se confondre, et renaître » met en scène un scénario baptismal dans lequel l'Idéal est à portée de main (1998b). Cet Idéal est "l'or du rêve ", celui du " Toast funèbre " des Poésies quand la « coupe vide " offerte laisse entrevoir un " monstre d'or». Cet or monstrueux et salutaire est celui de l'imaginaire en lequel le poète renaît en héros. L'opération «baptismale de lart » est encore plus manifeste dans le scénario marin de la " troupe de sirènes maintes à l'envers » de "Salut ». Mouvement de «torsion » qui éclate de toutes parts pour renaître encore ailleurs. Aussi, dans le célèbre poème des «Fenêtres ", le poète qui contemple son reflet dans une vitre, renaît dans l'œuvre :

Je me mire et me vois ange ! et je meurs, et jaime

Que la vitre soit l'art, soit la mysticité - ,

A renaître, portant mon rêve en diadème,

Au ciel antérieur où fleurit la Beauté ! 11

Sans le secours de l'art, la " Divinité de Soi " resterait inaccessible. Or l'ange qui naît dans le miroir de l’œuvre témoigne de létat de divinité intérieure, impersonnelle et ingénue. Comme le papier, dont Mallarmé évoque "l'ingénuité », vertu communément partagée entre les créateurs. Entendu qu' « appuyer selon la page, au blanc, inaugure son ingénuité, à soi $»^{12}$. D’ailleurs, chez Mallarmé le fond impersonnel de l'Être est ingénu et blanc, comme la page. L'enfance de l'art.

10. S. Mallarmé, La Musique et les Lettres, OC II, (2003: 74).

11. S. Mallarmé, «Les Fenêtres », Poésies, OC I, (1998: 104).

12. S. Mallarmé, La Musique et les Lettres, OC II, (2003: 234). 


\section{Traduire la Chimère, faire dans la dentelle}

Les hybrides mallarméens - par-delà la diversité de leurs figures - composent un monde commun et délicat : « il n'existe [...] rien qu'un compte exact de purs motifs rythmiques de lêtre [...] : il me plaît de les partout déchiffrer ». Ces structures, bien que restreintes communiquent entre-elles. Le nuage, l'écume, la plume, l'arabesque ou léventail, tous ces signifiants récurrents de la poétique mallarméenne se font signe d'abord entre eux, réalisant ainsi une singulière poétique de l'entrelacs. Nous dirons du schème de l'hybridité ce que Jean-Pierre Richard dit de la thématique : il « apparaît comme l'élément transitif qui nous permet de parcourir en divers sens toute l'étendue interne de l'ouvre, ou plutôt comme l'élément charnière grâce auquel elle s'articule en un volume signifiant. [Il] relève ainsi à la fois d'une cybernétique et d'une systématique " (1961 : 26). Réunies sous les auspices d'un même dynamisme - comme un balancement de lêtre - ces figurations hybrides semblent préparer toutes sortes de métamorphoses provisoires. Comme la série des éventails, ces amalgames d’ailes, de papiers, de voiles, de plis, de plumes, de sceptres ne se donnent, ne se réalisent que dans un souffle douverture et de fermeture, un battement ${ }^{13}$. Ce sont des schèmes dynamiques, des charnières poétiques. L'éventail, en ce sens, est l'illustration très schématique de cette articulation vitale. Il vaut comme pivot permettant la distribution des thèmes et des motifs. De la sorte, « des motifs de même jeu séquilibreront, balancés, à distance $»^{14}$. Ainsi, la danseuse devient une «métaphore résumant un des aspects élémentaires de notre forme, glaive, coupe, fleur, etc. ${ }^{15}$. Figure privilégiée de la Synthèse, « la vigueur charnelle et l'intégrité interne des tissus » (Richard, 1961 : 129) réalise un pur mouvement qui est l'Idée. Sans surprise, son balancement vital son chiffre - est identique à celui de la Chimère " tordue ", " relativement à la Loïe Fuller en tant qu'elle se propage, alentour, de tissus ramenés à sa personne, par l'action d'une danse $»^{16}$. Autre scène : dans Igitur, les meubles un peu danseurs « tordent » eux aussi « leurs chimères dans le vide ${ }^{17}$. Cette chimère permet certainement toutes les confusions de la rêverie (Smith, 1953 : 72), entendu qu'il s'agit au fond d' " y confondre selon des torsions le bizarre luxe de sa propre chimère ${ }^{18}$. Ainsi la musique est-elle d'abord une " chimère orchestrale ${ }^{19}$ présidée par un chef d'orchestre qui « détaille et contient la chimère $»^{20}$. Elle est semblable au théâtre dont « louverture de gueule de chimère [est] méconnue et frustrée à grand soin par l'arrangement

13. En particulier, Mattiussi (1998a).

14. S. Mallarmé, Crise de vers, OC II, (2003: 211).

15. S. Mallarmé, «Ballets », Crayonné au théâtre, Divagations, OC II, (2003 : 171).

16. S. Mallarmé, "Considérations sur l’art du ballet et la Loïe Fuller », Articles parus dans The National Observer, Dossier de "Divagations », OC II, (2003: 312).

17. S. Mallarmé, Igitur..., OC I, (1998: 864).

18. S. Mallarmé, Crayonné au théâtre, Divagations, OC II , (2003 : 183).

19. S. Mallarmé, «Laction restreinte ", Divagations, OC II, (2003: 216).

20. S. Mallarmé, «Plaisir sacré », Offices, OC II, (2003 : 237). 
social $»^{21}$. À la poésie, il revient de «traduire la Chimère $»^{22}$. Plus exactement, c'est la dévotion poétique qui permet de " boire à la source féconde de Chimère » quand le poète est « sylphe de ce froid plafond $»^{23}$. On pourrait multiplier à loisir les occurrences... Ces motifs, thèmes et figures intriqués témoignent de la même « communauté impossible » présidée par le schème de l'hybridité.

Madeleine Smith a relevé la jonction interculturelle des mythologies grecques, romaines et chinoises dans la figure de la Chimère, probablement héritière des Émaux et Camées de Théophile Gautier (Smith, 1953) : " Fais les sirènes bleues,/ Tordant de cent façons/ Leurs queues,/ Les monstres des blasons». Nulle surprise donc à ce que ce rêve s'empare du poète : "Un rêve m’étreint sous sa griffe/ Et j’ai, toujours sans dormir, beau/ Cajoler ce vain hippogriffe ${ }^{24}$. " Décor de l'absence », la poésie inaugure cette hybridité dans la "glace chimérique » du rêve. Le poète est lui-même un faune " accroché à la Chimère ". Par extension, le poème est le seul véritable " décor de l'hybridité $~^{25}$. Il faut donc moins s'attacher à y chercher un quelconque référent qu'identifier d'abord l'« ambiguïté de quelques figues belles, aux intersections $»^{26}$. De la même façon, le nuage, lécume, la plume, l'arabesque ou léventail ne valent que corrélés à une poétique généralisée de l’entrelacs. Aussi, la flûte Syrinx de L'Après-midi d'un faune, "instrument des fuites ${ }^{27}$ figure-t-elle l'attribut essentiel du poète : comme la dentelle, la plume ou l'écume, elle « retient l'infini, tissé par mille, chacun selon le fil ou prolongement ignoré son secret, assemble des entrelacs distants où dort un luxe à inventorier, stryge, nœud, feuillages $»^{28}$. Le poète lui-même est voué à cette articulation primordiale puisque "l'écrivain, de ses maux, dragons qu'il a choyés, ou d'une allégresse, doit s'instituer, au texte, le spirituel histrion $»^{29}$.

Dans le bestiaire mythopoétique de Mallarmé, la sirène ${ }^{30}$ est un doublet aquatique de la Chimère et de la danseuse. La danseuse elle-même n'est qu'une sirène mondaine " debout en sa torsion de sirène ». Elle se lie par analogie à lécume et à la beauté : "Vague d'azur Toi dont la blanche écume Fut mère de Vénus »" . Elle rejaillit en «fureur éparses en écumes ${ }^{32}$ autour de la danseuse. Dans "À la nue accablante... ",

21. S. Mallarmé, Crayonné au théâtre, Divagations, OC II, (2003: 161).

22. S. Mallarmé, «Le livre, instrument spirituel », Quant au livre, Divagations, OC II, (2003:226).

23. S. Mallarmé, «Surgi de la croupe... », Poésies, OC I, (1998 : 101).

24. S. Mallarmé, XXX, Vers de circonstance, OC I, (1998:324).

25. En particulier, Mattiussi (1998a).

26. S. Mallarmé, La Musique et les Lettres, OC II, (2003: 68).

27. S. Mallarmé, L’Après-midi d’un faune, OC I, (1998: 164).

28. S. Mallarmé, «L'Action restreinte », Quant au livre, Divagations, OC II, (2003 : 215).

29. Ibid.

30. La critique allemande s'est tout particulièrement intéressée à cette figure de la sirène, probablement grâce à la connivence qu'entretient le folklore avec ce mythe. La sirène se confond parfois avec Mélusine ou la Lorelei. On pense également à l'Ondine de Fouquet (1811). Voir aussi Schulze (1986: 54).

31. S. Mallarmé, "Chant d'ivresse ", Poèmes de jeunesse, OC I, (1998 : 187).

32. S. Mallarmé, « Billet à Whistler », Poésies, OC I, (1998:34). 
le " cheveu » d'écume noie le "flanc enfant d'une sirène " ${ }^{33}$. Danseuse, écume et sirène sont ainsi réunies sous les auspices mythopoétiques d'un même dynamisme ${ }^{34}$. Êtres doubles et ambigus, ils manifestent le même projet d'expansion et de métamorphose, peut-être même la même incrédulité tragique. Dans l'oscillation de son corps, la danseuse réalise «l'hymen » de l'humanité et de «l'éparse beauté générale ». Elle se dissipe en un éclat impalpable, aérien, presque immatériel comme ces robes de bal croquées dans La Dernière Mode : "La tradition, à laquelle plus ou moins obéissent toutes les Toilettes de Bal, je la définis : rendre légère, vaporeuse, aérienne pour cette façon supérieure de marcher qui s'appelle danser, la divinité apparue en leur nuage $»^{35}$. Sur ses pointes, déjà elle transgresse les espaces et franchit toutes sortes de seuils. Son mouvement étant analogue au "Signe " ou " hiéroglyphe ", elle se lie de complicité avec l'espace quelle strie, comme l'écume, le nuage, la dentelle, toutes ces matières aérées, trouées, subtiles que l'on retrouve fréquemment dans le corpus mallarméen, y compris au moment de désigner l'écriture poétique elle-même. Ainsi, comme le suggère l'Action restreinte, l'essentiel se joue peut-être bien « dans un présent qui joue à l'effacé pour couvrir de plus hybrides dessous " ${ }^{36}$, à côté des blancs ${ }^{37}$. Le texte, enfin, est arabesque, texture aérée, tissu troué, dentelle noire sur blanc.

\section{3. Être(s) né(s) de l'écume, aphrologie mallarméenne}

Arrivé à cette "ambiguïté même ", il faut entendre là où, en haute mer, le poème rejoint la sirène.

À la nue accablante tu

Basse de basalte et de laves

À même les échos esclaves

ar une trompe sans vertu

Quel sépulcral naufrage (tu

Le sais, écume, mais y baves)

Suprême une entre les épaves

Abolit le mât dévêtu

33. S. Mallarmé, «À la nue accablante... », Poésies, OC I, (1998 : 44).

34. En particulier, Richard, "Structure et Métaphore », (1961:411).

35. S. Mallarmé, La Dernière Mode, sixième livraison, 15 novembre 1874, OC II, (2003: 599).

36. S. Mallarmé, "Le Genre ou des modernes ", Crayonné au Théâtre, Divagations, OC II, (2003: 185).

37. En particulier, Mattiussi (1999: 174). 
Ou cela que furibond faute

De quelque perdition haute

Tout l'abîme vain éployé

Dans le si blanc cheveu qui traîne

Avarement aura noyé

Le flanc enfant d'une sirène ${ }^{38}$.

Publié le 15 mai 1894, dans la revue Obole littéraire, ce sonnet répond au «Salut » initial des Poésies sans pourtant indiquer la moindre réponse. Bertrand Marchal commente : "Si l'aventure poétique, à la manière d'une moderne odyssée, se développe ainsi entre l'écume initiale du vers ("Rien, cette écume vierge vers") et lécume finale d'une sirène noyée, cette aventure-là s'inscrit doublement dans l'espace du livre, entre la page blanche et le livre fermé, et peut un autre nom : la lecture $(\ldots) »^{39}$. De "Salut» à « À la nue accablante », en passant par « Brise marine » et " Au seul souci de voyager ", les Poésies achèvent ainsi un cycle marin dont la navigation intime est celle de l'œuvre et la sirène la trace d'un passage furtif et " rien au-delà ».

Sous un ciel inquiétant, lécume résiduelle semble divulguer un mystérieux naufrage mais aucun indice sinon ce peu d'écume ne vérifie ce drame - «naufrage total » pour reprendre l'expression de Maurice Blanchot (Hoppenot, 2006 : 139). Il faut en effet présupposer que le naufrage est sépulcral, " pour qu’il ait ainsi, comme un meurtre parfait, aboli jusqu’au mât, cette épave suprême, toujours dernière engloutie, plantée sur locéan comme la croix d'un cimetière marin " commente magistralement Bertrand Marchal (1985 : 253). Le poème est l'espace d'un « drame supposé » (ibid.) et pour ainsi dire virtuel, qui, comme dans le Coup de dés, agit " par raccourci, en hypothèse ». Ou bien cette mer échevelée, dentelle marine ne trahirait-elle - " faute d'une proie plus consistante " (ibid.) - que la noyade d'une sirène enfant, "sirène dont le cheveu blanchirait encore la vague furibonde " (ibid.) ? À la supposition de quelque "perdition haute » succèderait ainsi le drame d'une sirène moins engloutie qu'invisible. Or, ce naufrage est tu, occulté. À la lecture du premier vers, "À la nue accablante tu ", ce qui aiguise le plus l'activité interprétative, c'est le rapport qu'entretient le mot "nue » avec le participe passé « tu ». À l'absence de vêtement, si l’on concède la métaphore et non plus le simple nuage, répond l'absence de parole. « Tu » apparaît quatre fois à la rime, aux vers $1,4,5,8$ et toujours en des postures ambiguës car il pourrait tout aussi bien s'agir du participe passé du verbe taire, du pronom personnel « tu » que du verbe tuer. L'indécision sémantique encourage l'activité interprétative la plus dense.

Nous pouvons jouer avec les mots, Mallarmé lui-même nous y autorise souvent : la «trompe sans vertu » ne serait-elle pas moins une trompe sans force ni capacité

38. S. Mallarmé, « À la nue accablante tu », OC I, (1998: 44).

39. B. Marchal, « Notice des Poésies », OC I, (1998:1140). 
qu'une trompe sans vers tu, une trompe qui ne va pas du tout vers «tu »? Vers tu? Vers taire ? Comme un vers passé sous silence ? Taire le vers, dans ce cas, reviendrait-il à le tuer ? Ou faut-il tuer le vers pour que naisse le poème? Au vers 5 , «tu » continues à occuper une étrange position : il est à la rime et en début de parenthèse ; surtout, il est là où devrait être le participe passé «tu » du premier vers. Qu'est-ce donc alors qui est « tu » par cette trompe sans vertu ? Marchal et d'autres commentateurs ont remarqué que l'accord du participe passé révèle que ce n'est certes pas la nue qui est « tu » car il y manque le « e ». Comme c'est souvent le cas chez Mallarmé, il faut prêter une attention accrue au sujet du poème, dans tous les sens du terme. La sirène ne serait-elle pas, comme le faune ou la princesse de sang mêlée, Hérodiade, le poème lui-même ? Poème au mât aboli, comme dans « Brise marine » des Poésies ? Ou peutêtre ce naufrage n'est-il pas le désastre attendu mais bien plutôt le signe d'une glorieuse odyssée ? Bertrand Marchal l'interprète ainsi : "Car le naufrage n'est pas l'accident regrettable qui viendrait interrompre une aventure heureuse; le naufrage est à l'arrivée, comme il était déjà au départ, ou plutôt, pour mieux dire, il n’y a arrivée ni départ ; il n’y a, ainsi que le montrera le Coup de dés, qu'une immense tautologie, comme un tourbillon marin où s'abolit le temps fictif de lexistence $»^{40}$. Pris dans les contre-courants de la poésie, le temps de la sirène n'est plus le temps ordinaire, mais un espace imaginaire où l'envers de la présence retourne le poème, symbolisé par la sirène, tout aussi bien dans l'espace que dans le temps. Car le naufrage est à l'arrivée comme il est au départ, sans commencement ni fin. Naviguer ou sombrer, quelle différence s'il n'y a pas de port ? De ce point de vue, le dernier vers de « Toute lâme résumée... » propose une étrange rime inclusive faisant peut-être figure d'indice : " Le sens précis rature/ Ta vague littérature ${ }^{41}$. Lis tes ratures/la "vague littérature " faite d'une écume surnageant après la noyade des sirènes. Et partant, une étrange " corps et graphie » sous-marine. C’est aussi en ce sens que le poème est à interpréter comme l'écume d'un Rien. Un blanc. Cheveu, «tresse en flots » ${ }^{42}$, onde, dentelle saline, volute marine, ondine, sirène, naïade, vague... On sait que ce lexique nage de substitution en substitution précisément pour s'inscrire dans l'incertitude d'une vague et de façon à ce que nul ne puisse plus simplement jeter l'encre, pas même la sirène. Comme le voyage n'a pas de port, il n'y a pas plus d'amarre à larguer que d'amarre à atteindre, nul rivage des Phéaciens.

Chez Mallarmé, la figure hydride est promesse d'une eucharistie antique et païenne, emblème du poème lui-même, puissance d'un chant qui sait en même temps se faire entendre et se transformer en silence. Ainsi, comme ce toast plein des bulles de la poésie, l'apparition du poème ne conforte pas qu'une simple ivresse. Le poème, vaisseau fantôme parfois malmené par de pirates lecteurs, devient d'abord un silence

40. Ibid., p. 254.

41. S. Mallarmé, « Toute lâme résumée », OC I, (1998:60).

42. S. Mallarmé, « Le Château de l'espérance », Poèmes retrouvés, OC I, (1998: 65). 
intérieur. Comme l'enfant au coquillage, il fait tendre l'oreille à son propre silence. Comme Vénus sortie des flots, dont Mallarmé fait un symbole de la Poésie, le poème né de l'écume y retourne dans l'envers d'une sirène, proche du "Rien » (mais rien n'est pas rien), où tout se dissout en une " onde toi devenue » ${ }^{43}$, un " petit air » donc, qui estompe la différence entre tout pour élaborer une sorte d'unité entrelacée à ellemême. La queue de poisson figure la modernité poétique.

\section{BIBLIOGRAPHIE}

Barrère J-B. 1958. Hugo et le chèvre-pied. La Poésie d'inspiration païenne au XIX ${ }^{\mathrm{e}}$ siècle. Cahiers de l'Association internationale des études françaises $10 . \mathrm{n}^{\circ} 1.121-137$.

Belleau R. 1995. Euvres poétiques. Paris. Honoré Champion.

Derrida J. 1972. La Dissémination. Paris. Seuil.

Dubos J-B. 1770. Réflexions critiques sur la poésie et sur la peinture, t.1. Paris Pissot. $7^{\mathrm{e}}$ éd.

Hoppenot E. 2006. L’Épreuve du temps chez Maurice Blanchot. Paris. Éditions Complicités.

MallarméS. 1998. Euvres complètes, t. I.Éd. Bertrand Marchal. Paris. Gallimard. coll. «Bibliothèque de la Pléiade ».

Mallarmé S. 2003. Euvres complètes, t. II. Éd. Bertrand Marchal. Paris. Gallimard. coll. « Bibliothèque de la Pléiade ».

Marchal B. 1985. Lectures de Mallarmé. Poésies, Igitur, Le Coup de dés. Paris. José Corti.

Marchal B. 1988. La religion de Mallarmé : poésie, mythologie et religion. Paris. Corti.

Mattiussi L. 1998a. Emblèmes mallarméens de l'hybridité. Poétique 114. 141-159.

Mattiussi L. 1998b. Mallarmé et le procès d'impersonnification : Narcisse se dévisage. Romantisme 99. 105-116.

Mattiussi L. 1999. Figures du (re)créateur dans les Poésies. In Diaz J.-L. Les Poésies de Stéphane Mallarmé : "Une rose dans les ténèbres ». Société des études romantiques (Romantismel Colloques). Paris. SEDES. 171-180.

Mondor H. 1946. Vie de Mallarmé. Paris. Gallimard.

Nordau M. S. 1894. Dégénérescence. Paris. F. Alcan.

Richard J.-P. 1961. L'Univers imaginaire de Mallarmé. Paris. Seuil.

Schinz A. 1937. D’où sort l'Après-midi d’un faune de Mallarmé ? Modern Language Notes 52. Nº. 485-487.

Schulze J. 1986. Mallarmé najaden und Sirenen und das Sonett Petit Air I. Archiv für das Studium der neueren Sprachen und Literaturen 233. Erich Schmidt Verlag. 82-103.

Smith M. 1953. Mallarmé and the Chimères. Yale French Studies 11. 59-72.

\section{Mallarmé and poetics of hybridity}

ABSTRACT: The hybridity that is seen everywhere in symbolist poetry is not so much evidence of a temporary trend of the era following the interest in the crossing of species in nature as much as it is an attempt to confront the very nature of "art" itself. The

43. S. Mallarmé, « Petit Air I », OC I, (1998 : 35). 
time of the "quarrel of monsters" has passed, and the hybrids of contemporary poetry - competing for a place in the mythological scene that is symbolism - roam guiltless. And yet, beyond literary conventions, this mythological bestiary is anything but innocent and outdated; in fact, it embodies a genuine mythopoetic repertoire. For Mallarmé in particular, this hybrid bestiary reflects the illusive "Chimera" of poetry.

Keywords: Mallarmé, hybridity, modernity, symbolist poetry, mythological bestiary. 